\title{
Nondestructive cavity QED probe of Bloch oscillations in a gas of ultracold atoms
}

\author{
B. M. Peden, ${ }^{1}$ D. Meiser, ${ }^{1}$ M. L. Chiofalo, ${ }^{2}$ and M. J. Holland ${ }^{1}$ \\ ${ }^{1}$ JILA and Department of Physics, University of Colorado at Boulder, Colorado 80309-0440, USA \\ ${ }^{2}$ Department of Mathematics and INFN, University of Pisa, I-56100Pisa, Italy
}

(Received 3 June 2009; published 5 October 2009)

\begin{abstract}
We describe a scheme for probing a gas of ultracold atoms trapped in an optical lattice and moving in the presence of an external potential. The probe is nondestructive and uses the existing lattice fields as the measurement device. Two counterpropagating cavity fields simultaneously set up a conservative lattice potential and a weak quantum probe of the atomic motion. Balanced heterodyne detection of the probe field at the cavity output along with integration in time and across the atomic cloud yield information about the atomic dynamics in a single run. The scheme is applied to a measurement of the Bloch oscillation frequency for atoms moving in the presence of the local gravitational potential. Signal-to-noise ratios are estimated to be as high as $10^{4}$.
\end{abstract}

DOI: 10.1103/PhysRevA.80.043803

PACS number(s): 37.30.+i, 37.10.Jk, 03.75.Lm, 42.50.Ct

\section{INTRODUCTION}

The simulation of many-body models using gases of ultracold atoms trapped in optical lattices [1] has been successful in investigating many systems in condensed-matter physics. Band physics in gases of noninteracting Fermi gases in periodic potentials has been studied [2], quantum phase transitions such as the Mott insulator to superfluid transition have been observed [3], and strongly correlated physics such as in one-dimensional systems $[4,5]$ has been investigated. In these experiments, techniques such as time-of-flight measurements and Bragg spectroscopy are typically employed to probe atomic states and dynamics in optical lattices.

In this paper, we present an alternative method for optically probing atomic gases in optical lattices subjected to an external potential. The method is in situ and nondestructively measures properties of the atomic motion via weak coupling to the existing lattice fields. The technique satisfies three main goals. The probe is weak so that the atoms can be continuously monitored without affecting their dynamics; the existing lattice fields are employed as the probe, so that no external interrogation fields are necessary; and the signal-tonoise ratio (SNR) is large enough for experimental detection. In a ring cavity, two counterpropagating running-wave modes interact with a gas of ultracold atoms and simultaneously set up both a conservative external lattice potential for the atoms and a weak quantum optical probe of the atomic center-of-mass dynamics. The probe field leaks out of the cavity and is detected with a balanced heterodyne scheme at the cavity output.

This method is in a sense dual to strong measurement schemes such as time-of-flight absorption imaging and Bragg spectroscopy. In these schemes, light from a strong source is either absorbed by or scattered off of the atomic cloud. This allows for high-resolution images and a strong signal using only a single measurement, but the atomic sample is destroyed in the process. Here, the probe field is very weak so that a continuous measurement is made without affecting the atomic dynamics. Integration of the signal in time and across the atomic cloud yields measurements of dynamical properties of the atoms with a measurable SNR in a single experi- mental run at the price of losing information about individual atoms and real-time dynamics.

The procedure is similar in nature to recent proposals for optical detection of many-body atomic states. In one scheme, a weak probe beam is scattered off of atoms trapped in an optical lattice into a cavity mode, and signatures of manybody states such as Mott insulators and superfluids appear in the outcoupled fields [6]. In another, atoms in a lattice interact with two counterpropagating ring-cavity modes, and atomic number statistics can be inferred from the behavior of the cavity fields [7]. Bloch oscillations of atoms in a lattice can also be monitored via the transmission of light through a cavity [8].

Related techniques have been applied to nondestructive optical measurements of Rabi oscillations in gases of Cs atoms [9], of the Cs clock transition pseudospin [10], and of nonlinear dynamics in cold gases [11]. In addition, state preparation such as atomic spin squeezing via measurements on outcoupled cavity fields has been proposed [12-14]. It has also been demonstrated that the motion of individual atoms in an optical cavity can be tracked by the transmission of a probe field [15].

Here, we provide a test of the technique for the conceptually simple motion of noninteracting atoms in an optical lattice driven by a constant force, which leads to Bloch oscillations [16]. This choice is motivated by the fact that Bloch oscillations can be viewed as a probe for investigating quantum gases in optical lattices. These oscillations may be used in the measurement of fundamental constants [17], to provide levels of precision up to $\delta g / g \approx 10^{-7}$ in the measurement of the acceleration of gravity [18-21], and to measure Casimir forces on small length scales [22]. When interactions are significant, damping of Bloch oscillations provide information on correlation-induced relaxation processes [23-27]. Finally, this investigation is a starting point for other measurement schemes, such as periodically driven lattices acting as a spectroscopic probe of atomic motion [21].

The paper is organized as follows. In Sec. II, we present the details of the system and detection scheme. In Sec. III, we apply this scheme to the detection of Bloch oscillations in an optical lattice. In Sec. IV, we summarize the main results of the paper and conclude with prospects for measurements 
of many-body properties of gases of ultracold atoms trapped in optical lattices.

\section{MODEL AND DETECTION SCHEME}

We briefly outline the physical basis of the model and approximations used. This includes a discussion of obstacles in the way of satisfying the goals outlined above, avoiding these problems, and the conditions required for the method to work.

To set up a conservative lattice potential, many photons must be present in the cavity field, so that fluctuations can be neglected, and this necessitates a strong pumping from the incoupled lasers. On the other hand, the probe field amplitude must be small enough, so that it does not affect the atomic dynamics, requiring a weak pumping. In addition, the probe and lattice fields couple to each other through the scattering of photons off of the atoms. This acts as an extra source for probe dynamics. The probe field is then not a direct measure of atomic dynamics and can act back on the atoms, altering the properties we are attempting to measure.

We can circumvent these problems by first choosing the relative phase on the incoupled lasers, so that only one of two standing-wave modes in the cavity is pumped. Strong pumping and the properties of a bad cavity - where the fields are at all times in steady state-ensure that the pumped mode acts as a lattice potential. The other standing-wave mode is not pumped. Any field leaking out of the cavity from this mode arises solely because of events occurring in the cavity, and it can therefore act as a probe for system dynamics.

Two conditions must be met in order to ensure that the nonpumped mode acts as a probe of atomic dynamics. The probe must have as its source only the motion of the atoms. This means that any probe dynamics due to the effective coupling to the lattice field must be small compared to that induced by the motion of the atoms. The back-action of this mode on the atoms must also be negligible. Any atomic motion induced by coupling to the probe field must be small compared to the motion induced by both lattice and external potentials.

Two conditions are also required for the pumped field to act as a conservative lattice potential. The back-action of the atoms on the lattice field must be negligible, meaning that deviations from the mean-field amplitude caused by coupling to the atoms are small compared to the mean-field amplitude itself. In addition, any atomic motion induced by fluctuations away from the mean lattice field must be small compared to that induced by the external potential, since this is the interesting dynamics.

The scheme is realized in the setup illustrated in Fig. 1. We consider $N_{a}$ ground-state atoms interacting with two counterpropagating running-wave cavity modes in a ring resonator setup. The two modes of the cavity have frequency $\omega_{c a}$ and wave vectors $\pm k_{c a} \hat{z}$. The two cavity modes are coherently pumped at a detuning $\Delta_{p}=\omega_{L}-\omega_{c a}$, where $\omega_{L}$ is the frequency of the pumping lasers. Photon decay through the cavity mirrors is treated within the Born-Markov approximation. We treat the atom-cavity-field interaction in rotatingwave and dipole approximations. The cavity modes are far-

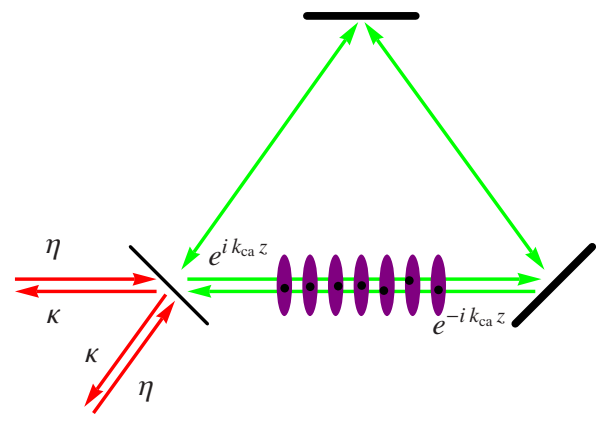

FIG. 1. (Color online) Schematic of the coupled atom-cavity system. Incoupled lasers set up two counterpropagating fields within the cavity. The atoms interact with the cavity fields via the optical dipole potential. Photons from the cavity beams exit the cavity through the mirrors at a rate $\kappa$.

detuned from atomic transitions. This same setup has been used in a cooling scheme for atoms [28].

\section{A. Model}

The effective Hamiltonian for the coupled atom-cavity system is given by

$$
\begin{aligned}
\hat{H}= & \int d z \hat{\Psi}^{\dagger}(z)\left(-\frac{\hbar^{2}}{2 m} \frac{d^{2}}{d z^{2}}+V_{e x t}(z)\right) \hat{\Psi}(z) \\
& +\sum_{k= \pm k_{c a}}\left(\hbar \eta \hat{a}_{k}+\hbar \eta \hat{a}_{k}^{\dagger}-\hbar \Delta_{p} \hat{a}_{k}^{\dagger} \hat{a}_{k}\right) \\
& +\int d z \hat{\Psi}^{\dagger}(z) \hbar g_{0}\left(\hat{a}_{k_{c a}}^{\dagger} e^{-i k_{c a} z}+\hat{a}_{-k_{c a}}^{\dagger} e^{i k_{c a} z}\right) \\
& \times\left(\hat{a}_{k_{c a}} e^{i k_{c a} z}+\hat{a}_{-k_{c a}} e^{-i k_{c a} z}\right) \hat{\Psi}(z) .
\end{aligned}
$$

Here, $\hat{\Psi}$ is the atomic field operator and $\hat{a}_{k}$ is the annihilation operator for the cavity mode $k$. The parameter $m$ is the mass of the atom, $g_{0}$ is the two-photon atom-cavity coupling, and $\eta$ is the strength of the cavity field pumping, taken to be real. Due to the far detuning of the cavity fields from the atomic transition, excited states of the atom have been adiabatically eliminated, and the atoms couple to the field intensity. The three terms in Eq. (1) are, respectively, the atomic kinetic energy and external potential, the bare cavity mode Hamiltonian, and the atom-cavity interaction. Cavity losses through the cavity mirrors are treated via a master equation with Liouvillian,

$$
\hat{\mathcal{L}} \hat{\rho}=-\frac{\hbar \kappa}{2} \sum_{k= \pm k_{c a}}\left(\hat{a}_{k}^{\dagger} \hat{a}_{k} \hat{\rho}+\hat{\rho} \hat{a}_{k}^{\dagger} \hat{a}_{k}-2 \hat{a}_{k} \hat{\rho} \hat{a}_{k}^{\dagger}\right),
$$

where $\hat{\rho}$ is the reduced density matrix for the atom-cavity system and $\kappa$ is the cavity linewidth.

We perform a canonical transformation of the cavity mode operators to symmetric and antisymmetric modes,

$$
\hat{b}_{ \pm}=\frac{\hat{a}_{k_{c a}} \pm \hat{a}_{-k_{c a}}}{\sqrt{2}} .
$$

The bare cavity Hamiltonian in terms of these operators is given by 


$$
\hat{H}_{c a}=\sqrt{2} \hbar \eta\left(\hat{b}_{+}+\hat{b}_{+}^{\dagger}\right)-\hbar \Delta_{p}\left(\hat{b}_{+}^{\dagger} \hat{b}_{+}+\hat{b}_{-}^{\dagger} \hat{b}_{-}\right) .
$$

The symmetric mode $\hat{b}_{+}$is pumped by the incoupled lasers, whereas the antisymmetric mode $\hat{b}_{-}$is not. The $\hat{b}_{+}$mode has a mode function proportional to $\cos \left(k_{c a} z\right)$ and sets up the lattice potential as follows.

The equation of motion for the symmetric field amplitude is

$$
i \frac{d\left\langle\hat{b}_{+}\right\rangle}{d t}=\left(-i \frac{\kappa}{2}-\Delta_{p}\right)\left\langle\hat{b}_{+}\right\rangle+\sqrt{2} \eta+2 g_{0}\left\langle\hat{b}_{+} \hat{C}\right\rangle+i g_{0}\left\langle\hat{b}_{-} \hat{S}_{2}\right\rangle,
$$

where

$$
\begin{aligned}
& \hat{C}=\int d z \cos ^{2}\left(k_{c a} z\right) \hat{\Psi}^{\dagger}(z) \hat{\Psi}(z), \\
& \hat{S}_{2}=\int d z \sin \left(2 k_{c a} z\right) \hat{\Psi}^{\dagger}(z) \hat{\Psi}(z) .
\end{aligned}
$$

We perform another transformation to a fluctuation operator, $\hat{d}_{+}=\hat{b}_{+}-\beta$, leaving the antisymmetric mode unchanged, $\hat{d}_{-}$ $=\hat{b}_{-}$. The mean steady-state amplitude $\beta$ is given by

$$
\beta=\left\langle\hat{b}_{+}\right\rangle=\frac{\sqrt{2} \eta}{\Delta_{p}-2 g_{0}\langle\hat{C}\rangle_{t=0}+i \kappa / 2},
$$

where the expectation value is evaluated at time $t=0$.

The equations of motion for both $\hat{d}_{+}$and $\hat{d}_{-}$are given by

$$
i \frac{d\left\langle\hat{d}_{-}\right\rangle}{d t}=\left(-\Delta_{p}-i \frac{\kappa}{2}\right)\left\langle\hat{d}_{-}\right\rangle+2 g_{0}\left\langle\hat{d}_{-} \hat{S}\right\rangle-i g_{0}\left\langle\hat{d}_{+} \hat{S}_{2}\right\rangle-i g_{0} \beta\left\langle\hat{S}_{2}\right\rangle
$$

and

$$
\begin{aligned}
i \frac{d\left\langle\hat{d}_{+}\right\rangle}{d t}= & \left(-\Delta_{p}-i \frac{\kappa}{2}\right)\left\langle\hat{d}_{+}\right\rangle+2 g_{0}\left\langle\hat{d}_{+} \hat{C}\right\rangle+i g_{0}\left\langle\hat{d}_{-} \hat{S}_{2}\right\rangle \\
& +2 g_{0} \beta\left(\langle\hat{C}\rangle-\langle\hat{C}\rangle_{t=0}\right)
\end{aligned}
$$

where

$$
\hat{S}=\int d z \sin ^{2}\left(k_{c a} z\right) \hat{\Psi}^{\dagger}(z) \hat{\Psi}(z)
$$

Finally, the equation of motion for the atomic field operator is given by

$$
\begin{aligned}
i \hbar \frac{d \hat{\Psi}(z)}{d t}= & \left(-\frac{\hbar^{2}}{2 m} \frac{d^{2}}{d z^{2}}+V_{l a t}(z)+V_{e x t}(z)\right) \hat{\Psi}(z) \\
& +i \hbar g_{0}\left(\beta^{*} \hat{d}_{-}-\beta \hat{d}_{-}^{\dagger}+\hat{d}_{+}^{\dagger} \hat{d}_{-}-\hat{d}_{-}^{\dagger} \hat{d}_{+}\right) \sin \left(2 k_{c a} z\right) \hat{\Psi}(z) \\
& +2 \hbar g_{0} \hat{d}_{-}^{\dagger} \hat{d}_{-} \sin ^{2}\left(k_{c a} z\right) \hat{\Psi}(z)+2 \hbar g_{0}\left(\hat{d}_{+}^{\dagger} \hat{d}_{+}+\beta \hat{d}_{+}^{\dagger}\right. \\
& \left.+\beta^{*} \hat{d}_{+}\right) \cos ^{2}\left(k_{c a} z\right) \hat{\Psi}(z),
\end{aligned}
$$

where $V_{\text {lat }}(z)=V_{0} \cos ^{2}\left(k_{c a} z\right)$ is a conservative lattice potential of depth $V_{0}=2 \hbar g_{0}|\beta|^{2}$.
Upon examining Eqs. (9) and (10), it is apparent that, in order for $\hat{d}_{-}$to act as a probe field, we must be able to neglect the higher-order atom-field correlations such as $\left\langle\hat{d}_{+} \hat{S}_{2}\right\rangle$. In addition, we must be able to neglect all but the first term in Eq. (12) in order that the lattice field act as an external conservative potential. In the correlation hierarchy, each consecutive order scales as $\left|g_{0}\right| N_{a} / \kappa$ times the previous order. For instance, $\left\langle\hat{d}_{+} \hat{\Psi}^{\dagger} \hat{\Psi}\right\rangle$ scales as $\left|g_{0}\right| N_{a} / \kappa$ times $\left\langle\hat{\Psi}^{\dagger} \hat{\Psi}\right\rangle$. Therefore, requiring $\left|g_{0}\right| N_{a} / \kappa \ll 1$ allows us to neglect all terms involving correlations between three or more operators. Solving Eqs. (9) and (10) by adiabatically slaving the fields to the atomic motion then yields

$$
\begin{gathered}
\left\langle\hat{d}_{-}\right\rangle=\frac{-i g_{0} \beta\left\langle\hat{S}_{2}\right\rangle}{\Delta_{p}+i \kappa / 2}, \\
\left\langle\hat{d}_{+}\right\rangle=\frac{2 g_{0} \beta\left(\langle\hat{C}\rangle-\langle\hat{C}\rangle_{t=0}\right)}{\Delta_{p}+i \kappa / 2} .
\end{gathered}
$$

This implies that the effective couplings in Eq. (12) such as $g_{0} \hat{d}_{+}^{\dagger} \hat{d}_{+}$scale as $V_{0}\left(g_{0} N_{a} / \kappa\right)^{2}$. In turn, this implies that the dominant terms on the right-hand side of Eq. (12) are the atomic kinetic energy and the lattice potential. All terms involving cavity field operators can be neglected to first order. Ensuring that we can neglect these terms compared to the external potential requires explicit knowledge of the form of $V_{e x t}$ and will therefore be left for the next section. Finally, we point out that, while these are necessary conditions, finding the exact criteria for neglecting the back-action of the probe on the atoms and the atoms on the lattice requires a more careful analysis of the problem, including numerical simulations. This is left for future work.

With these approximations in hand, the equation of motion for the atomic field operator is

$$
i \hbar \frac{d \hat{\Psi}(z)}{d t}=\left(-\frac{\hbar^{2}}{2 m} \frac{d^{2}}{d z^{2}}+V_{l a t}(z)+V_{e x t}(z)\right) \hat{\Psi}(z),
$$

and the probe field amplitude is given by

$$
\left\langle\hat{d}_{-}(t)\right\rangle=\frac{-i g_{0} \beta}{\Delta_{p}+i \kappa / 2}\left\langle\hat{S}_{2}\right\rangle,
$$

where we have approximately solved Eq. (9) by adiabatically slaving $\left\langle\hat{d}_{-}\right\rangle$to the atomic motion. Since the atoms are independent and couple symmetrically to the light field, we can in Eq. (16) make the replacement

$$
\left\langle\hat{S}_{2}\right\rangle \rightarrow N_{a}\left\langle\psi(t)\left|\sin \left(2 k_{c a} \hat{z}\right)\right| \psi(t)\right\rangle,
$$

where $|\psi(t)\rangle$ is a single-particle state satisfying Eq. (15).

\section{B. Detection scheme}

Through Eq. (16), $\left\langle\hat{d}_{-}\right\rangle$provides a measure of the atomic dynamics within the cavity. In [13], two schemes for detection of atomic motion using the outcoupled cavity fields were presented. Here, we briefly review the superior case, where heterodyne detection of $\hat{d}_{-}$is performed by beating the field 


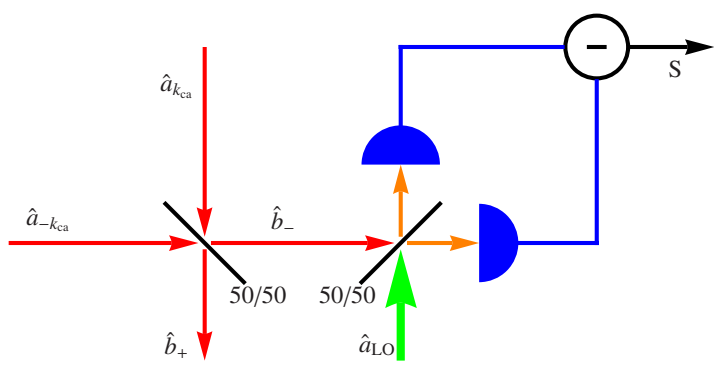

FIG. 2. (Color online) Schematic of the balanced heterodyne detection scheme. The outcoupled cavity beams $\hat{a}_{ \pm k_{c a}}$ are combined to form symmetric $\left(\hat{b}_{+}\right)$and antisymmetric $\left(\hat{b}_{-}\right)$modes. The antisymmetric mode beats against a strong $\mathrm{LO}, \hat{a}_{\mathrm{LO}}$, and photodetectors count the number of photons in the quadratures of $\hat{b}_{-}$. The difference of these counts is the signal.

against a strong local oscillator (LO), as illustrated in Fig. 2.

The field at the output of the cavity is a combination of the outcoupled lattice and probe fields, the pump field, and the reflected light from the pump field. Since we have chosen to pump only the lattice mode, the probe mode is orthogonal to the other three contributions to this field. The outcoupled light from the probe can then be separated out by means of a beam splitter. According to input-output theory [29], this field is proportional to

$$
\hat{d}_{\text {out }}=\sqrt{\kappa} \hat{d}_{-}+\hat{d}_{\text {in }} .
$$

By beating this field against a strong local oscillator, these photons can be detected with unit efficiency. The input field state is the vacuum, in which case $\left\langle\hat{d}_{\text {out }}\right\rangle=\sqrt{\kappa}\left\langle\hat{d}_{-}\right\rangle$. The resulting signal is the difference signal at the output of the photodetectors, given by

$$
V(t) \propto \operatorname{Im}\left[\sqrt{\kappa} \alpha_{\mathrm{LO}_{0}}^{*}\left\langle\hat{d}_{-}\right\rangle(t)\right],
$$

which is a product of $\left|\alpha_{\mathrm{LO}}\right|$ with

$$
\hat{q}_{-}=e^{-i \phi_{\mathrm{LO}}} \hat{d}_{-}-e^{i \phi_{\mathrm{LO}}} \hat{d}_{-}^{\dagger},
$$

which is a quadrature of the antisymmetric mode field. The local oscillator amplitude is $\alpha_{\mathrm{LO}}=\left|\alpha_{\mathrm{LO}}\right| e^{i \phi_{\mathrm{LO}}}$. The SNR is the ratio of the signal power to signal variance, given by

$$
\mathrm{SNR}=\int d \omega\left|\sqrt{\kappa}\left\langle\hat{q}_{-}\right\rangle(\omega)\right|^{2} .
$$

The integrand is proportional to the power spectrum $S(\omega)$ of the signal current in Eq. (19).

\section{RESULTS}

In this paper, we consider the motion of atoms confined in the optical lattice in the presence of gravity,

$$
V_{\text {ext }}(z)=m g z
$$

and use the scheme outlined in the previous section to probe the motion of the atoms. Gravity measurements are important, for instance, for optical lattice clocks [30]. For this reason, we treat the specific system of a gas of ${ }^{87} \mathrm{Sr}$ atoms, although the method certainly applies to many species of atoms. The parameters for the coupled atom-cavity system are chosen to reflect current experimental conditions. They are $\lambda_{c a}=2 \pi / k_{c a}=813 \mathrm{~nm}, \hbar \kappa=100 E_{R}, \Delta_{p}=0, \hbar g_{0}=10^{-4} E_{R}$, and $N_{a}=10^{4}$, implying derived parameters of $E_{R} \approx(2 \pi$ $\times 4 \mathrm{kHz}) \hbar$ and $m g d \approx 0.25 E_{R} ; E_{R}=\hbar^{2} k_{c a}^{2} / 2 m$ is the recoil energy of the lattice; and $d=\pi / k_{c a}$ is the lattice spacing.

We have to ensure that the back-action of both $\hat{d}_{-}$and $\hat{d}_{+}$ on the atoms is still negligible. Specifically, the coupling strengths in Eq. (12) must be small compared to the characteristic coupling strength of $V_{\text {ext }}, \hbar \omega_{B}=m g d$. These conditions are met if $\left|V_{0}\right|\left(g_{0} N_{a} / \kappa\right)^{2} \ll \hbar \omega_{B}$. This inequality is satisfied for the parameters above. Again, while these conditions are necessary, the exact criteria for being able to neglect the back-action of the fields on the atoms requires more careful numerical study, which will be left for future work.

Within this setup, we envision an experiment in which the atoms are initially loaded into a harmonic trap. A vertical one-dimensional optical lattice is slowly ramped on, so that the atoms are in the ground state of the combined potential of trap and lattice for a noninteracting gas. The trap is switched off over a time that is long compared to the time scale of vibrational dynamics in order to prevent interband transitions during the switch-off. The gas is then allowed to evolve under gravity. In the presence of such a constant force, the atoms undergo Bloch oscillations. This dynamics is briefly reviewed in the following discussion.

\section{A. System dynamics}

The central result of the theory describing Bloch oscillations is based on a semiclassical equation of motion [16], which states that the average quasimomentum of a wave packet restricted to the first band increases linearly in time until it reaches the Brillouin-zone (BZ) boundary, at which point it is Bragg reflected. Explicitly, this is

$$
\hbar\langle q\rangle(t)=\hbar\langle q\rangle(0)+m g t,
$$

where the quasimomentum $q$ is restricted to the range $-k_{c a}$ $\leq q \leq k_{c a}$. Since the group velocity of the atomic wave packet is given by the derivative of the dispersion relation [16], the periodic nature of the quasimomentum implies that the atomic momentum oscillates at a frequency $\omega_{B}=m g d / \hbar$. These Bloch oscillations will persist as long as there is negligible Landau-Zener tunneling to higher bands. Each time the wave packet reaches the BZ boundary, a fraction of population is transferred to the second band, given by [31]

$$
P_{L Z}=\exp \left(-\frac{\pi^{2}}{8} \frac{\Delta^{2}}{m g d E_{R}}\right),
$$

where $\Delta$ is the band gap at the boundary. When $\Delta^{2}$ $<4 m g d E_{R}$, the population transfer is appreciable, and vibrational dynamics significantly alter the behavior of the atoms. For this reason, we restrict our attention to lattice depths greater than $3 E_{R}$, where $P_{L Z}$ is at most $10^{-5}$ for our choice of parameters.

In order to understand how Bloch oscillations are reflected in the time dependence of the probe field, we care- 
fully consider Eq. (16). The operator $\sin \left(2 k_{c a} \hat{z}\right)$ is periodic in space with period $d$ and has odd parity, implying that it connects two Bloch states, $\left|\psi_{q}^{(n)}\right\rangle$ and $\left|\psi_{q^{\prime}}^{\left(n^{\prime}\right)}\right\rangle$, only if the quasimomenta are equal, $q=q^{\prime}$, and the bands satisfy $n-n^{\prime}=$ odd. Taking

$$
|\psi\rangle=\sum_{n, q} c_{q}^{(n)}\left|\psi_{q}^{(n)}\right\rangle
$$

we can write the matrix element in Eq. (16) approximately as

$$
\left\langle\psi\left|\sin \left(2 k_{c a} \hat{z}\right)\right| \psi\right\rangle \approx \sum_{q} \rho_{q, q}^{(1,2)}+\text { H.c. },
$$

where $\rho_{q, q}^{(1,2)}=c_{q}^{(1) *} c_{q}^{(2)}$ is the coherence between bands 1 and 2. This assumes an initial state confined to the first band in the case of negligible coupling to bands 3 and higher. Using Eq. (15), we can derive an approximate equation of motion for the coherence; it is

$$
i \frac{d \rho_{q, q}^{(1,2)}}{d t}=\Delta_{q}^{(1,2)} \rho_{q, q}^{(1,2)}+\omega_{B} \rho_{q}^{(1)},
$$

where $\rho_{q}^{(1)}$ is the population of the $q$-quasimomentum state in the first band and $\hbar \Delta_{q}^{(1,2)}=E_{q}^{(2)}-E_{q}^{(1)}$ is the energy difference between the $q$-quasimomentum Bloch states in the first two bands. Since $\Delta_{q}^{(1,2)} \gg \omega_{B}$, the coherence follows the first-band population adiabatically. In this approximation, $\rho_{q, q}^{(1,2)}$ $=-\rho_{q}^{(1)} \omega_{B} / \Delta_{q}^{(1,2)}$.

Combining this expression for a wave packet that is narrow in quasimomentum with Eq. (23), Eq. (16) approximately becomes

$$
\left\langle\hat{d}_{-}(t)\right\rangle \approx \frac{i g_{0} \beta N_{a}}{\Delta_{p}+i \kappa / 2} \frac{\omega_{B}}{\Delta_{m g t}^{(1,2)}} .
$$

This expression implies that the probe field amplitude is largest when the atomic wave packet is centered at the BZ boundary since $\Delta_{m g t}^{(1,2)}$ is smallest at this point.

Equations (15) and (16) are numerically integrated for an initial state that is a Gaussian of spatial width $\sigma$ projected into the first band. This approximates the ground state of the combined potential of lattice and harmonic trap for a noninteracting gas. An example of the system dynamics is illustrated in Fig. 3, where $V_{0}=-3 E_{R}$ and $\sigma=2 d$. A vertical slice through Fig. 3(a) is the wave-function density in the first band plotted versus quasimomentum at an instant in time. The center of this wave packet moves linearly in time and is reflected at the BZ boundary $\left(q=k_{c a}\right)$, as in Eq. (23). Bloch oscillations are illustrated in Fig. 3(b), where the atomic momentum oscillates in time. Finally, the response of the probe field to this dynamics is illustrated in Fig. 3(c). As predicted above, the probe field intensity peaks when the atomic wave packet reaches the BZ boundary.

\section{B. Signal and SNR}

As described in Sec. II, the probe field is combined at the cavity output with a strong local oscillator, and the resulting signal is proportional to a quadrature of the probe field [Eq. (20)]. An example of such a signal is plotted in Fig. 4. There (a)

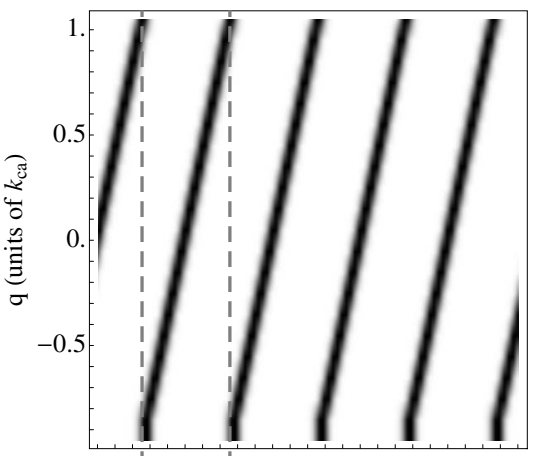

(b)
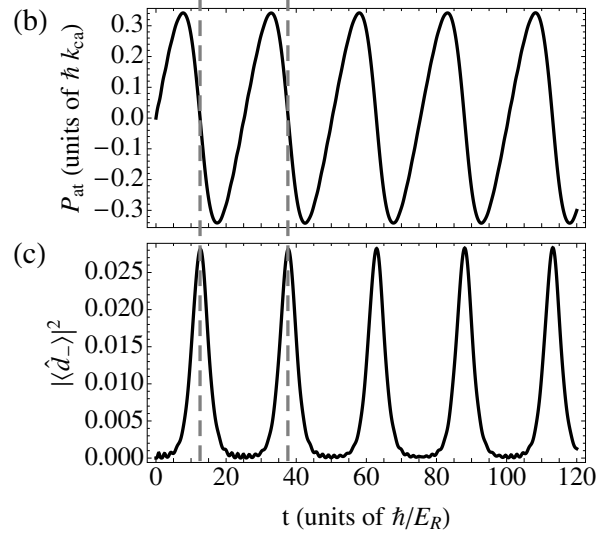

FIG. 3. Example of system dynamics for $V_{0}=-3 E_{R}$ and initial state a Gaussian of width $\sigma=2 d$ projected into the first band. (a) Atomic density in the first band plotted versus quasimomentum. White corresponds to zero population, black to maximal population. Population in the second band is at most $0.001 N_{a}$. (b) Expectation value of atomic momentum illustrating Bloch oscillations. (c) Number of photons in the probe field.

is a clear peak at the Bloch oscillation frequency in the signal power spectrum, but there are also several harmonics present. In calculating the SNR [Eq. (21)], we place a notch filter about $\omega_{B}$ and count only the total number of photons outcoupled from the quadrature at this frequency.

(a)
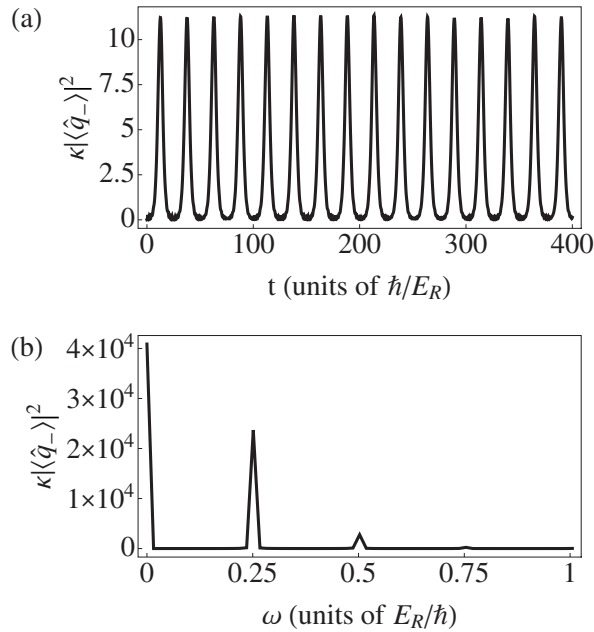

FIG. 4. Examples of the (a) signal power and (b) signal power spectrum, computed with parameters $V_{0}=-3 E_{R}, \hbar g_{0}=10^{-4} E_{R}, N_{a}$ $=10^{4}$, and $\hbar \kappa=100 E_{R}$. The signal displays a clear oscillation at the Bloch frequency $\omega_{B}=0.25 E_{R} / \hbar$. 


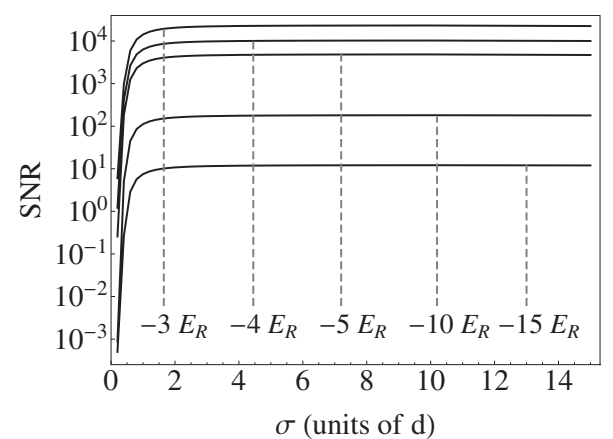

FIG. 5. Signal-to-noise ratio as a function of the initial wavepacket width for an interrogation time of $1 \mathrm{~s}$ and for lattice depths of $V_{0}=-3 E_{R},-4 E_{R},-5 E_{R},-10 E_{R},-15 E_{R}$. For $\sigma<2 d$, the SNR is reduced due to parts of the wave packet constantly moving past the Brillouin-zone boundary, where the signal peaks. The SNR saturates near $\sigma=2 d$.

There are a few properties of the system that can affect the SNR. First, the width of the initial wave packet has an effect. It is easiest to see why this is so by taking as the initial state a Wannier function, which is a coherent superposition of Bloch states in a single band, populated equally. According to Eq. (23), the wave packet is continuously reaching the BZ boundary, and the oscillation in the signal is washed out. Second, when the lattice is too deep, the first two bands are essentially flat, in which case $\Delta_{q}^{(1,2)}$ does not change with quasimomentum, eliminating the oscillations in the signal according to Eq. (28).

The temperature of the atomic gas can also significantly influence the SNR. For a finite temperature, the pure state in Eq. (17) is replaced with a density matrix. The incoherent sum over different states of the atom will alter the expectation value, possibly reducing it. In addition, the temperature and the chemical potential of the gas determine the relative populations of the various Bloch states, and appreciable population in higher bands can destroy Bloch oscillations. As long as the temperature is smaller than the band gap, $k_{B} T$ $\ll \hbar \Delta_{k_{c a}}^{(1,2)}$, this effect will be small.

Finally, the replacement, Eq. (17), cannot be made when the atoms are not independent, e.g., if they interact with each other. In this case it is possible that the scaling of the SNR with the number of atoms will be reduced from $N_{a}^{2}$. A proper treatment of thermal and interaction effects is necessary for exact results. Here, we neglect both interactions and finite temperature for the case of ${ }^{87} \mathrm{Sr}$ atoms, in which case Eq. (17) is valid.

Equation (12) is numerically integrated for a time $t$ $=400 \hbar / E_{R}$. The resulting wave function is used to compute the probe field amplitude [Eq. (16)], which is Fourier transformed and squared, yielding the power spectrum. The SNR is computed and scaled up linearly to an interrogation time of $1 \mathrm{~s}$, which assumes that coherence time of the Bloch oscillations is longer than $1 \mathrm{~s}$.

The results are plotted in Figs. 5 and 6. The SNR climbs from zero for small wave-packet widths and saturates near $\sigma=2 d$. The decrease in SNR for $\sigma<2 d$ is a result of the fact that the wave packet is wide in quasimomentum, which means that a significant portion of the wave packet is at the

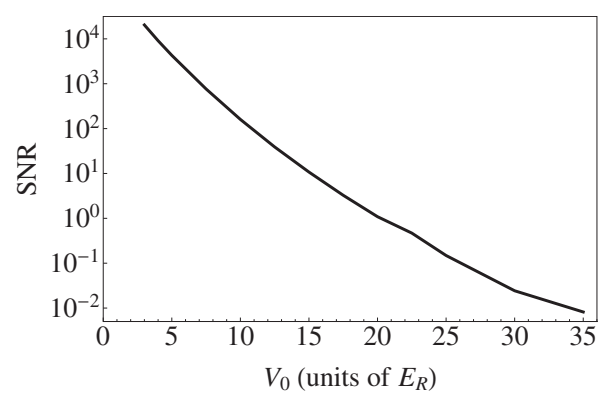

FIG. 6. Signal-to-noise ratio as a function of lattice depth for $\sigma=2 d$ and an interrogation time of $1 s$. For $\left|V_{0}\right|<3 E_{R}$, significant Landau-Zener tunneling to the second band destroys Bloch oscillations. For $\left|V_{0}\right|>3 E_{R}$, the SNR decreases due to the increasing flatness of the lowest band, which in turn decreases the amplitude in momentum space of the Bloch oscillations.

BZ boundary for all times. We get a maximum when the lattice depth is relatively small, $\left|V_{0}\right| \approx 3 E_{R}$, and the SNR decreases with increasing lattice depth.

\section{CONCLUSION}

We have described a general cavity QED system in which properties of atomic dynamics can be probed in situ and nondestructively. One cavity field is strong enough to act as a conservative lattice potential for the atoms, and the other cavity field is weak so that it acts as a nondestructive probe of atomic motion. This technique is applied to the detection of Bloch oscillations. Balanced heterodyne detection of the probe field at the cavity output combined with integration in time and across the atomic cloud allows for SNRs as high as $10^{4}$.

Examining Eqs. (16) and (21), we can see that the SNR can be increased by either decreasing the cavity linewidth $\kappa$ at fixed lattice depth and atom-cavity coupling or increasing the coupling constant $g_{0}$ at fixed $V_{0}$ and $\kappa$. The linewidth can be increased as long as the system remains in the bad cavity limit. However, a linewidth of $\hbar \kappa=100 E_{R}$ is already very small from an experimental standpoint, so increasing it beyond this level is a technological challenge. On the other hand, $g_{0}$ can be varied merely by varying the detuning between the cavity fields and atomic transitions. In addition, the SNR scales with the square of the number of atoms, so increasing $N_{a}$ beyond the $10^{4}$ level assumed in this paper is also desirable. This can all be done to the extent that the conditions outlined in Secs. II and III are still met.

This scheme can be extended for use in detection of various atomic properties, and the measurement of Bloch oscillations itself can be viewed as a general dc probe for atomic dynamics and states. For instance, Bloch oscillations may be used for measurement of fundamental constants [17] and for Casimir forces [22]. Varying the detuning between two lattice beams gives rise to an effective acceleration of the lattice [31], and band physics may be probed by varying the Bloch oscillation frequency in such a setup. Breakdown of Bloch oscillations are a signature of many-body effects in an atomic gas [23], and this is signaled by a reduction in SNR compared to the noninteracting case. 
Two generalizations of this measurement technique are readily realizable. We may implement a periodic forcing whose varying driving frequency can be a spectroscopic probe of atomic dynamics. The simplest examples of this include shaking the lattice [21] and modulating the amplitude of the lattice [32]. Another important extension of the method involves measuring higher-order correlation functions of the outcoupled probe field. Since one cavity field operator couples to two atomic field operators [see, for instance, Eq. (9)], higher-order properties of the atoms such as density-density correlations can easily be measured with standard quantum optical techniques. The use of higher-order correlation functions of the probe field is a starting point for generalizing this technique to probe many-body physics in optical lattices.

\section{ACKNOWLEDGMENTS}

We acknowledge useful conversations with Jun Ye, Ana Maria Rey, and Victor Gurarie. This work was supported by DOE, NSF, DFG (D.M.), and ASI Grant No. WP4200 (M.L.C.).
[1] D. Jaksch, C. Bruder, J. I. Cirac, C. W. Gardiner, and P. Zoller, Phys. Rev. Lett. 81, 3108 (1998).

[2] M. Köhl, H. Moritz, T. Stöferle, K. Günter, and T. Esslinger, Phys. Rev. Lett. 94, 080403 (2005).

[3] M. Greiner, O. Mandel, T. Esslinger, T. W. Hansch, and I. Bloch, Nature (London) 415, 39 (2002).

[4] B. Paredes, A. Widera, V. Murg, O. Mandel, S. Fölling, I. Cirac, G. V. Shlyapnikov, T. W. Hänsch, and I. Bloch, Nature (London) 429, 277 (2004).

[5] T. Stöferle, H. Moritz, C. Schori, M. Köhl, and T. Esslinger, Phys. Rev. Lett. 92, 130403 (2004).

[6] I. B. Mekhov, C. Maschler, and H. Ritsch, Phys. Rev. Lett. 98, 100402 (2007).

[7] W. Chen, D. Meiser, and P. Meystre, Phys. Rev. A 75, 023812 (2007).

[8] B. Prasanna Venkatesh, M. Trupke, E. A. Hinds, and D. H. J. O’Dell, e-print arXiv:0811.3993.

[9] P. J. Windpassinger, D. Oblak, P. G. Petrov, M. Kubasik, M. Saffman, C. L. Garrido Alzar, J. Appel, J. H. Muller, N. Kjaergaard, and E. S. Polzik, Phys. Rev. Lett. 100, 103601 (2008).

[10] S. Chaudhury, G. A. Smith, K. Schulz, and P. S. Jessen, Phys. Rev. Lett. 96, 043001 (2006).

[11] G. A. Smith, S. Chaudhury, A. Silberfarb, I. H. Deutsch, and P. S. Jessen, Phys. Rev. Lett. 93, 163602 (2004).

[12] A. E. B. Nielsen and K. Mølmer, Phys. Rev. A 77, 063811 (2008).

[13] D. Meiser, J. Ye, and M. Holland, New J. Phys. 10, 073014 (2008).

[14] I. B. Mekhov and H. Ritsch, Phys. Rev. Lett. 102, 020403 (2009).

[15] C. J. Hood, T. W. Lynn, A. C. Doherty, A. S. Parkins, and H. J. Kimble, Science 287, 1447 (2000).
[16] M. Ben Dahan, E. Peik, J. Reichel, Y. Castin, and C. Salomon, Phys. Rev. Lett. 76, 4508 (1996).

[17] P. Cladé, E. de Mirandes, M. Cadoret, S. Guellati-Khélifa, C. Schwob, F. Nez, L. Julien, and F. Biraben, Phys. Rev. Lett. 96, 033001 (2006).

[18] B. P. Anderson and M. A. Kasevich, Science 282, 1686 (1998).

[19] G. Roati, E. de Mirandes, F. Ferlaino, H. Ott, G. Modugno, and M. Inguscio, Phys. Rev. Lett. 92, 230402 (2004).

[20] I. Carusotto, L. Pitaevskii, S. Stringari, G. Modugno, and M. Inguscio, Phys. Rev. Lett. 95, 093202 (2005).

[21] V. V. Ivanov, A. Alberti, M. Schioppo, G. Ferrari, M. Artoni, M. L. Chiofalo, and G. M. Tino, Phys. Rev. Lett. 100, 043602 (2008).

[22] P. Wolf, P. Lemonde, A. Lambrecht, S. Bize, A. Landragin, and A. Clairon, Phys. Rev. A 75, 063608 (2007).

[23] J. K. Freericks, Phys. Rev. B 77, 075109 (2008).

[24] P. Mehta and N. Andrei, Phys. Rev. Lett. 96, 216802 (2006).

[25] T. Oka and H. Aoki, Phys. Rev. Lett. 95, 137601 (2005).

[26] A. Buchleitner and A. R. Kolovsky, Phys. Rev. Lett. 91, $253002(2003)$

[27] W. S. Dias, E. M. Nascimento, M. L. Lyra, and F. A. B. F. de Moura, Phys. Rev. B 76, 155124 (2007).

[28] A. Griessner, D. Jaksch, and P. Zoller, J. Phys. B 37, 1419 (2004).

[29] C. W. Gardiner and P. Zoller, Quantum Noise (Springer-Verlag, Berlin, 2004).

[30] A. D. Ludlow et al., Science 319, 1805 (2008).

[31] O. Morsch, J. H. Müller, M. Cristiani, D. Ciampini, and E. Arimondo, Phys. Rev. Lett. 87, 140402 (2001).

[32] A. Alberti, G. Ferrari, V. V. Ivanov, M. L. Chiofalo, and G. M. Tino, e-print arXiv:0903.0724. 